\title{
AN ANALOGUE OF THE LAW OF THE ITERATED LOGARITHM
}

\section{GLEN BAXTER}

1. Introduction. The following two theorems are well known:

Theorem A. Let $\left(X_{k}\right)(k=1,2, \cdots)$ be a sequence of independent, identically distributed Bernoulli variables with $P\left(X_{k}=1\right)=p$ and $P\left(X_{k}=0\right)=q$, and let $S_{n}=X_{1}+X_{2}+\cdots+X_{n}$. Then,

$$
\begin{gathered}
\lim _{n \rightarrow \infty} P\left\{\frac{S_{n}-n p}{(n p q)^{1 / 2}} \leqq \alpha\right\}=\left(\frac{1}{2 \pi}\right)^{1 / 2} \int_{-\infty}^{\alpha} e^{-t^{2} / 2} d t, \\
P\left\{\limsup _{n \rightarrow \infty} \frac{S_{n}-n p}{(2 n p q \log \log n)^{1 / 2}}=1\right\}=1 .
\end{gathered}
$$

TheOREM B. Let $\left(X_{n k}\right)(k=1, \cdots, n ; n=1,2, \cdots)$ be a sequence of sequences of Bernoulli variables, independent and identically distributed within each row, with $P\left(X_{n k}=1\right)=\lambda / n$ and $P\left(X_{n k}=0\right)$ $=1-\lambda / n$, and let $S_{n}=X_{n 1}+X_{n 2}+\cdots+X_{n n}$. Then,

$$
\lim _{n \rightarrow \infty} P\left\{S_{n} \leqq \alpha\right\}=\sum_{k \leqq \alpha} e^{-\lambda} \frac{\lambda^{k}}{k !} .
$$

In view of Theorem $A$, Theorem $B$ does not seem complete for the analogue of (2) is missing. In this paper we develop for Theorem B two possible analogues of (2) each arising from certain additional (but,nonoverlapping) conditions on the sequence of sequences $\left(X_{n k}\right)$. In the following we shall understand that $\left(X_{n k}\right)$ satisfies the conditions of Theorem $B$ and that

$$
S_{n}=X_{n 1}+X_{n 2}+\cdots+X_{n n} .
$$

2. Preliminary results. To facilitate the proofs in the next section we state here two preliminary results. First, let an integer $a>\lambda$ be given and let $A_{n}$ be the event $S_{n} \geqq a$. Then

$$
P\left\{A_{n}\right\}=\sum_{k \geqq a}^{n}\left(\frac{\lambda}{n}\right)^{k}\left(1-\frac{\lambda}{n}\right)^{n-k}{ }_{n} C_{k}
$$

so that

Presented to the Society, September 3, 1954; received by the editors July 12, 1954. 


$$
\begin{aligned}
\frac{\lambda^{a}}{a !} e^{-\lambda}\left\{1-\frac{a(a-1)}{2 n}\right\} & <\left(\frac{\lambda}{n}\right)^{a}{ }_{n} C_{a} e^{-\lambda}<P\left\{A_{n}\right\} \\
& <\left(\frac{\lambda}{n}\right)^{a}{ }_{n} C_{a} \frac{1}{1-\lambda / a}<\frac{\lambda^{a}}{a !} \frac{1}{1-\lambda / a} .
\end{aligned}
$$

Second, if $a_{n}$ is the greatest integer in $c \log n / \log \log n$, where $c$ is any positive real number, then

$$
\sum_{n=10}^{\infty} \frac{\lambda^{a_{n}}}{a_{n} !}
$$

converges or diverges according as $c>1$ or $c<1$. For, by Stirling's formula

$$
\frac{\lambda^{a_{n}}}{a_{n} !} \sim\left(\frac{1}{2 \pi a_{n}}\right)^{1 / 2}\left(\frac{\lambda e}{a_{n}}\right)^{a_{n}}
$$

and the question of convergence or divergence of the summation

$$
\sum_{n=10}^{\infty}\left(\frac{1}{2 \pi a_{n}}\right)^{1 / 2}\left(\frac{\lambda e}{a_{n}}\right)^{a_{n}}
$$

can easily be reduced to that of the integral

$$
\int_{e^{2}}^{\infty}\left(\frac{\log \log x}{2 \pi c \log x}\right)^{1 / 2}\left(\frac{\lambda e \log \log x}{c \log x}\right)^{c \log x / \log \log x} d x
$$

$$
=\int_{2}^{\infty}\left(\frac{\log y}{2 \pi c y}\right)^{1 / 2}\left(\frac{\lambda e \log y}{c y^{1-1 / c}}\right)^{c y / \log y} d y .
$$

A consequence of the second result is the following: let $\mu$ be any real number greater than 1 , let $n_{r}$ be the greatest integer in $\mu^{r}$ $(r=1,2, \cdots)$, and let $b_{r}$ be the greatest integer in

$$
c \log \log n_{r} / \log \log \log n_{r}
$$

Then

$$
\sum_{r=100}^{\infty} \frac{\lambda^{b r}}{b_{r} !}
$$

converges or diverges according as $c>1$ or $c<1$.

3. The analogues. The first result we obtain arises from the assumption that all of the variables $\left(X_{n k}\right)$ are independent.

THEOREM 1. If $\left(X_{n k}\right)$ is a sequence of sequences of independent ran- 
dom variables (satisfying the conditions of Theorem 2), then

$$
P\left\{\limsup _{n \rightarrow \infty} \frac{\log \log n}{\log n} S_{n}=1\right\}=1 \text {. }
$$

Proof. The proof follows the usual lines, employing the BorelCantelli lemmas. Let $a_{n}$ be the greatest integer in $c \log n / \log \log n$, where $c$ is a positive real number, and let $A_{n}$ be the event $S_{n} \geqq a_{n}$. Since the events $A_{1}, A_{2}, \cdots$ are independent, it is sufficient in proving the theorem to show that the summation

$$
\sum_{n=10}^{\infty} P\left\{A_{n}\right\}
$$

converges or diverges according as $c>1$ or $c<1$. By the estimates in (5) we reduce this to showing that the summation

$$
\sum_{n=10}^{\infty} \frac{\lambda^{a_{n}}}{a_{n} !}
$$

converges or diverges according as $c>1$ or $c<1$. The identity of (13) and (6) then finishes the proof.

An obvious difference between Theorems A and B is that A relates to a sequence of random variables while $B$ relates to a sequence of sequences of random variables. It is true that Theorem $A$ may be rephrased so that it also relates to a sequence of sequences, but in this rephrasing very definite connections arise between random variables in different rows. Certainly, these connections are important for any "strong law" result like (2). In Theorem 1 connections (complete independence) had to be postulated before any result analogous to (2) could be obtained. It may be noted, however, that the condition of Theorem 1 does not correspond to the "natural" conditions which arise in rephrasing Theorem A to sequence of sequences. The rather strange sounding conditions of the next theorem are motivated by the desire for this correspondence.

Theorem 2. If $\left(X_{n k}\right)$ satisfies

(a) the variables in any column $\left(X_{k k}, X_{k+1, k}, X_{k+2, k}, \cdots\right)$ are independent of the variables in any other column, then

(b) $X_{k k} \geqq X_{k+1, k} \geqq X_{k+2, k} \geqq \cdots$,

$$
P\left\{\limsup _{n \rightarrow \infty} \frac{\log \log \log n}{\log \log n} S_{n}=1\right\}=1
$$


Proof. Let a real number $\mu>1$ be given, let $n_{r}$ be the greatest integer in $\mu^{r}(r=1,2, \cdots)$, and let $b_{r}$ be the greatest integer in $c \log \log n_{r} / \log \log \log n_{r}$, where $c$ will be specified later.

We first show that if $c>1$, then with probability one only finitely many of the following events occur: $S_{k} \geqq b_{r}$ for at least one $k$ with $n_{r} \leqq k<n_{r+1}$. By hypothesis (b) and the fact that $X_{n k}$ is non-negative, it is sufficient to show that with probability one only finitely many of the events

$$
S_{n_{r}}+X_{n_{r}+1, n_{r}+1}+X_{n_{r}+2, n_{r}+2}+\cdots+X_{n_{r+1}, n_{r}+1} \geqq b_{r}
$$

occur. If we call this event $B_{r}$, then

$$
\begin{aligned}
P\left\{B_{r}\right\} & <\sum_{k \geqq b_{r}}^{n_{r}+1}\left(\frac{\lambda}{n_{r}}\right)^{k}\left(1-\frac{\lambda}{n_{r+1}}\right)^{n_{r+1}-k}{ }_{n_{r+1}} C_{k} \\
& =\sum_{k \geqq b_{r}}^{n_{r+1}}\left(\frac{n_{r+1} \lambda / n_{r}}{n_{r+1}}\right)^{k}\left(1-\frac{\lambda}{n_{r+1}}\right)^{n_{r+1}-k}{ }_{n_{r+1}} C_{k} \\
& <\left(\frac{\lambda n_{r+1}}{n_{r}}\right)^{b_{r}} \frac{1}{b_{r} !} \frac{1}{1-\lambda / n_{r}}
\end{aligned}
$$

by the estimates in (5). Since $n_{r+1} / n_{r} \sim \mu$, the result follows as an application of the first Borel-Cantelli lemma and of (10) where $\lambda$ is replaced by $\lambda \mu$.

We next show that if $c<1$, then with probability one infinitely many of the following events occur: $S_{k} \geqq b_{r+1}$ for at least one $k$ with $n_{r} \leqq k<n_{++1}$. Because of hypothesis (b) and the fact that $X_{n k}$ is nonnegative it is sufficient to show that with probability one infinitely many of the events

$$
X_{n_{r+1}, n_{r-1}+1}+X_{n_{r+1}, n_{r-1}+2}+\cdots+X_{n_{r+1}, n_{r}} \geqq b_{r+1}
$$

occur. If we call this event $C_{r}$, then $C_{1}, C_{2}, \cdots$ are independent and for large values of $r$

$$
\begin{aligned}
P\left\{C_{r}\right\} & =\sum_{k \geqq b_{r+1}}^{n_{r}-n_{r}-1}\left(\frac{\lambda}{n_{r+1}}\right)^{k}\left(1-\frac{\lambda}{n_{r+1}}\right)^{n_{r}-n_{r-1}-k}{ }_{n_{r}-n_{r-1}} C_{k} \\
& >\left(\frac{\lambda}{n_{r+1}}\right)^{b_{r+1}} e^{-\lambda}{ }_{n_{r}-n_{r-1}} C_{b_{r+1}} \\
& >\frac{\lambda^{b_{r+1}}}{b_{r+1} !} e^{-\lambda}\left(\frac{n_{r}}{n_{r+1}}\right)^{b_{r+1}}\left(1-\frac{b_{r}^{2}}{n_{r}}\right) .
\end{aligned}
$$

Since $n_{r+1} / n_{r} \sim \mu$, the result follows as an application of the second 
Borel-Cantelli lemma and of (10) where $\lambda$ is replaced by $\lambda / \mu$. This finishes the proof.

It is interesting to note that although $\lambda$ appears in the definition of $X_{n k}$ and in (3), it does not appear in (11) or (14).

CORNELl UNiversity AND

MassachusetTs InStitute of TEChNOLOGY

\section{FAMILIES OF PERIODIC SOLUTIONS OF SYSTEMS HAV- ING RELATIVELY INVARIANT LINE INTEGRALS ${ }^{1}$}

D. C. LEWIS

The purpose of this paper is to generalize to all systems having relatively invariant line integrals a known theorem about Lagrangian (or Hamiltonian) systems of differential equations. This known theorem is roughly to the effect that in any given family of periodic solutions the period is at most a function of the energy constant alone; that is, it does not depend upon the parameters of the family except in so far as the energy is a function of these parameters. An exception may occur, however, if the energy itself is independent of the parameters.

The history of this particularly elegant theorem began over eighty years ago, but the only presentation in a standard treatise known to me is in the book of A. Wintner, Analytical foundations of celestial mechanics, Princeton University Press, 1941, pp. 73, 74, and p. 414, where reference to the older literature is to be found. The only flaw in this treatment is the omission of mention of the exception noted above in case the energy constant is independent of the parameters. A satisfactory treatment in this respect is given by G. Herglotz, among the collection of papers by various authors published in book form: Probleme der Astronomie; Festschrift für Hugo v. Seeliger, Berlin, 1924, pp. 197-199.

The generalization given in this paper (cf. below the statement of the main theorem) is a bit more than a mere extension of the classical theorem to the Pfaffian equations of Birkhoff (which, by a suitable transformation on the dependent variables, can be written in Hamiltonian form, at least in the neighborhood of a given periodic solu-

Received by the editors June 14, 1954.

1 This research was supported by the United States Air Force through the Office of Scientific Research. 